\title{
Evaluación de usabilidad en herramientas de aprendizaje colaborativo en dispositivos móviles para ambientes virtuales educativos
}

\author{
Assessment of Usability in Collaborative Learning Tools in Mobile Devices \\ for Virtual Educational Environments
}

\begin{abstract}
Avaliação de usabilidade em ferramentas de aprendizagem colaborativa em dispositivos móveis para ambientes virtuais educacionais
\end{abstract}

\author{
Miguel Angel Couoh Novelo \\ Tecnológico Nacional de México / Instituto Tecnológico de Tizimín \\ miguelcouoh@gmail.com \\ https://orcid.org/0000-0001-8490-1954
}

\section{Resumen}

La educación superior actual incorpora cada vez más el uso de dispositivos tecnológicos y, con esto, las aplicaciones móviles resultan de suma importancia en este contexto. En este estudio se buscó evaluar el grado y los parámetros específicos de usabilidad de una aplicación móvil enfocada al trabajo colaborativo en el Instituto Tecnológico de Tizimín. Para determinar la muestra, se aplicó la fórmula de muestreo aleatorio estratificado. La recolección de datos se obtuvo a partir de tres salones de la carrera de ingeniería Informática: un total de 68 alumnos. Se aplicó un cuestionario que constó de 18 preguntas de opción múltiple y preguntas abiertas. La información se procesó mediante el software de estadística IBM SPSS. Los resultados arrojan que los alumnos cuentan con un sistema operativo, en su mayoría Android. Asimismo, prefieren que las aplicaciones sean gratuitas para el aprendizaje en la escuela. Además, consideran que en las asignaturas se deben emplear actividades que incluyan dispositivos móviles como estrategia de aprendizaje, ya que sería una ventaja. La aplicación cumple de manera positiva con las variables de usabilidad descritas, ya que 


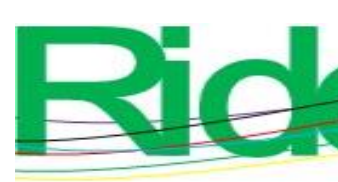

Revista Iberoamericana para la
Investigación y el Desarrollo Educativo
ISSN $2007-7467$

contiene lo necesario para un correcto funcionamiento en cuanto a diseño y usabilidad. Finalmente, se cumplió con la mayoría de los parámetros de usabilidad.

Palabras clave: aprendizaje en línea, enseñanza superior, evaluación de la tecnología.

\section{Abstract}

Today's higher education increasingly incorporates the use of technological devices and, with this, mobile applications are of utmost importance in this context. This study sought to evaluate the degree and specific usability parameters of a mobile application focused on collaborative work at the Instituto Tecnológico de Tizimín. To determine the sample, the stratified random sampling formula was applied; the data collection was obtained from three classrooms of the Computer Engineering career: a total of 68 students. A questionnaire was applied that consisted of 18 multiple-choice questions and open questions. The information was processed using the IBM SPSS statistical software. The results show that the students have an operating system, mostly Android. They also prefer the apps to be free for learning at school. In addition, they consider that in the subjects activities that include mobile devices should be used as a learning strategy, since it would be an advantage. The application complies in a positive way with the described usability variables, since it contains what is necessary for a correct operation in terms of design and usability. Finally, most of the usability parameters were met.

Keywords: online learning, higher education, technology assessment.

\section{Resumo}

O ensino superior de hoje incorpora cada vez mais a utilização de dispositivos tecnológicos e com isso as aplicações móveis assumem importância neste contexto. Este estudo procurou avaliar o grau e parâmetros específicos de usabilidade de uma aplicação móvel síncrona focada no trabalho colaborativo em sala de aula no Instituto Tecnológico de Tizimín. Para determinar a amostra, foi aplicada a fórmula de amostragem aleatória estratificada; A coleta de dados foi obtida a partir de três salas da carreira de Engenharia da Computação com um total de 68 alunos, onde foi aplicado um questionário composto por 18 questões de múltipla escolha e questões abertas. As informações foram processadas nos softwares estatísticos IBM SPSS (Statistical Product and Service Solutions) e no programa Microsoft Excel para Windows. Os resultados mostram que os alunos possuem um sistema operacional, 


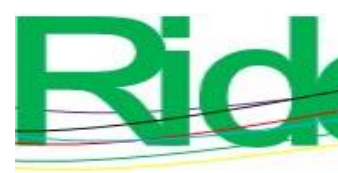

Revista Iberoamericana para la
Investigación y el Desarrollo Educativo
ISSN $2007-7467$

principalmente Android. Eles também preferem que os aplicativos sejam gratuitos para aprender na escola. Além disso, consideram que atividades que incluam dispositivos móveis como estratégia de aprendizagem devem ser utilizadas nas disciplinas, pois isso seria uma vantagem. A aplicação cumpre de forma positiva as variáveis de usabilidade descritas, visto que contém o que é necessário para um correto funcionamento em termos de design e usabilidade. Finalmente, a maioria dos parâmetros de usabilidade foram atendidos.

Palavras-chave: aprendizagem online, ensino superior, avaliação de tecnologia.

Fecha Recepción: Noviembre 2020

Fecha Aceptación: Mayo 2021

\section{Introducción}

El trabajo colaborativo dentro del aula no garantiza que todos los integrantes participen ni que se logre el objetivo de la actividad. Asimismo, el docente no cuenta con alguna herramienta que permita saber quién colaboró y quién no lo hizo. Lo anterior puede provocar dificultades al equipo de trabajo, así como al docente al momento de asignar una calificación.

Actualmente, la mayoría, si no es que todos los estudiantes, cuenta con un dispositivo móvil, y lo utilizan la mayor parte del tiempo. Teniendo en cuenta este factor, se desarrolló una app, una herramienta colaborativa mediante la cual los estudiantes podrán realizar de manera síncrona la actividad marcada en el aula, cada uno desde su dispositivo móvil.

El Instituto Tecnológico de Tizimín, ubicado en la ciudad de Tizimín, Yucatán, ofrece las carreras de Gestión Empresarial, Administración de Empresas, Biología, Agronomía e Informática. Esta última fue donde se realizó el presente estudio.

Así pues, el objetivo fue desarrollar una aplicación móvil que promoviera el trabajo colaborativo en los estudiantes de ingeniería Informática, carrera que imparte el Instituto Tecnológico de Tizimín. De igual manera, evaluar el grado y los parámetros específicos de usabilidad que deberá contener la aplicación móvil en modo síncrona. 


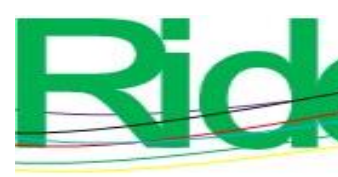
Revista Iberoamericana para la
Investigación y el Desarrollo Educativo
ISSN $2007-7467$

\section{Marco teórico}

El Joint Committee on Standards for Educational Evaluation señala que "la evaluación es el enjuiciamiento sistemático de la validez o mérito de un objeto" (Stufflebeam y Shinkfield, 1995, p. 19). Es importante considerar tanto lo bueno como lo malo de la situación evaluada, de lo contrario no se trata de una evaluación (Stufflebeam y Shinkfield, 1995). Una evaluación puede servir para medir la usabilidad de algo.

Actualmente, los usuarios tienen la opción de escoger entre una gran variedad de dispositivos móviles, los cuales ofrecen una amplia gama de aplicaciones para todo tipo de propósitos que agilizan los procesos cotidianos. Por ejemplo, las tabletas, que se asemejan a una minicomputadora del tamaño de un cuaderno pequeño, no necesitan periféricos para su utilización, ya que incorpora la tecnología touchscreen (Quisi, 2012).

Dentro de las aplicaciones móviles podemos encontrar incluso algunas para lograr un mejor aprendizaje

El aprendizaje colaborativo se caracteriza por el hecho de que los alumnos trabajan conjuntamente para ayudarse en la resolución de problemas, el intercambio de información, la producción de conocimientos y la mejora de la comunicación social. Además de estas contribuciones, la colaboración tiene ventajas motivacionales e intelectuales (Escofet y Marimon, 2012).

\section{Calidad de software}

La calidad del software es una combinación deseada de atributos. Dicha combinación deberá ser claramente especificada. Definir la calidad de software de un sistema equivale a definir una lista de atributos requeridos para ese sistema. Utilizando esta definición, se puede afirmar que lo indispensable es medir más puntualmente los atributos relacionados con la calidad. Dentro de estos atributos, uno de los considerados más importantes es la usabilidad, que indica la facilidad con la que un usuario puede hacer uso de una aplicación de software. Por lo tanto, resulta de interés poder obtener una medida del grado de usabilidad que tiene una aplicación (Institute of Electrical and Electronics Engineers [IEEE], 1998). 

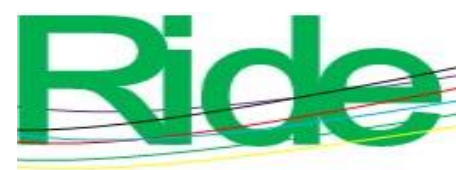

Revista Iberoamericana para la Investigación y el Desarrollo Educativo

ISSN $2007-7467$

Los modelos de usabilidad esencialmente están formados por la aplicación que se va a evaluar y la interacción que el usuario tiene con esta para alcanzar sus objetivos. La aplicación tiene definidos atributos y métricas asociadas para medir dichos atributos. Estos componentes se encuentran definidos dentro de un cierto contexto de uso, tal como se aprecia en la figura 1 (Enriquez y Casas, 2013).

Figura 1. Modelo de usabilidad

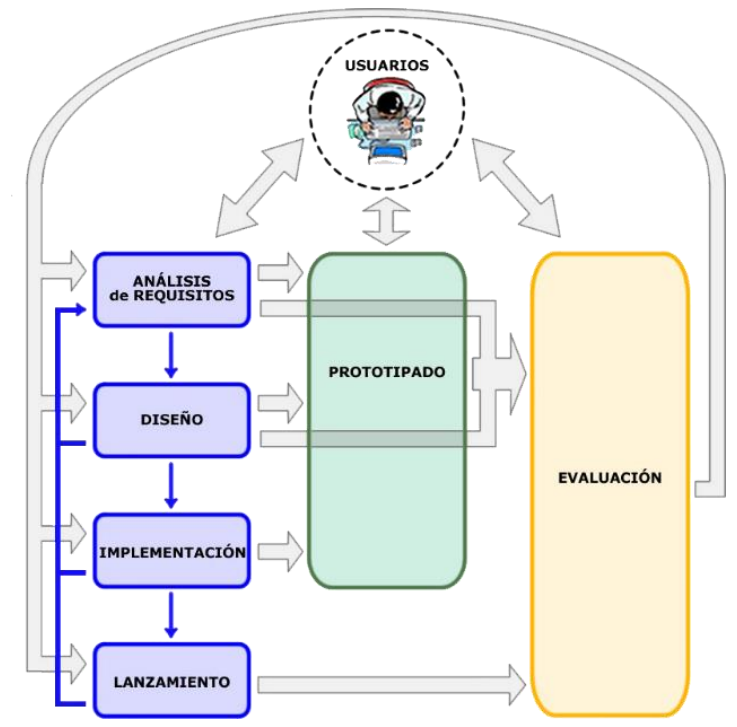

Fuente: Enriquez y Casas (2013)

Es importante recalcar que en la presente investigación se evaluó la usabilidad de una aplicación colaborativa desarrollada específicamente para dispositivos móviles, por lo que a continuación se visualizarán temas relacionados a esta tecnología.

\section{Dispositivos y aplicaciones móviles}

La definición de dispositivo móvil suele carecer de claridad. A continuación, presentamos la de Tardáguila (2009), que consideramos no adolece de tal defecto:

Aquellos microordenadores que son lo suficientemente ligeros como para ser transportados por una persona, y que disponen de la capacidad de batería suficiente como para poder funcionar de forma autónoma. Normalmente, son versiones limitadas en prestaciones, y por tanto en funcionalidades, de los ordenadores portátiles o de sobremesa (p. 4). 


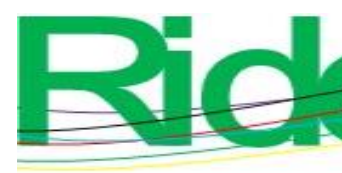

Revista Iberoamericana para la Investigación y el Desarrollo Educativo ISSN $2007-7467$

\section{Aplicaciones y herramientas síncronas y asíncronas}

La comunicación sincrónica es el intercambio de información por Internet en tiempo real y la comunicación asincrónica se establece entre dos o más personas de manera diferida en el tiempo, es decir, cuando no existe coincidencia temporal (Lamí, Pérez y Rodríguez del Rey, 2016, p. 85).

\section{Aprendizaje colaborativo}

El aprendizaje colaborativo es un proceso de construcción social en el que cada profesional aprende más de lo que aprendería por sí mismo debido a la interacción con otros miembros de su comunidad profesional o de su grupo de estudio. Lo que antes era una clase, se convierte en un foro abierto al diálogo entre estudiantes o entre estudiantes, profesores y tutores. Los estudiantes ahora participan activamente en situaciones interesantes y demandantes.

\section{Objetivo principal}

La presente investigación tiene como objetivo principal evaluar el grado y los parámetros específicos de usabilidad que deberá contener la aplicación móvil enfocada al trabajo colaborativo dentro del aula de clase.

\section{Preguntas de investigación}

A partir de lo anterior se generó la siguiente pregunta de investigación: ¿la aplicación móvil cumple con los parámetros y grado de usabilidad para funcionar de manera pertinente en un ambiente educativo virtual? Y las preguntas que se probaron para medirla son: ¿cada pantalla contiene un título o encabezado que describe el contenido? ¿El diseño de los íconos tiene relación con la función del sistema? Cuando se selecciona un ícono, ¿se distingue el ícono seleccionado claramente? ¿Considera necesario informar al usuario cuando debe de esperar algún procedimiento del sistema? ¿Los íconos que se manejan en el sistema son de fácil familiarización para el usuario? ¿El lenguaje que se utiliza es claro? ¿Se pregunta al usuario que confirme acciones que tendrán consecuencias drásticas, es decir, como eliminar datos? ¿Existe alguna opción en el sistema que permita a los usuarios regresar al menú anterior? ¿Existe algún elemento que permita al usuario identificar la ventana en la que se encuentra? ¿Considera adecuado que los mensajes de error generen sonidos de alerta? ¿Los 


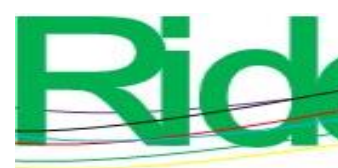

Revista Iberoamericana para la Investigación y el Desarrollo Educativo ISSN $2007-7467$

mensajes de error son breves y adecuados? ¿Los mensajes de error son apropiados y respetuosos para los usuarios? ¿Los mensajes de error están gramaticalmente correctos? Si se detecta un error en una caja de texto, ¿el sistema posiciona el cursor en esa misma caja? ¿Los mensajes de error indican qué acción debe realizar el usuario para corregir el problema? ¿Las cajas de texto indican el límite de letras que se pueden ingresar? ¿El sistema muestra cuando una caja de texto está inactiva? ¿Las cajas de texto que son opcionales están claramente marcadas? ¿Los colores utilizados dentro del sistema son consistentes? ¿Existe buen contraste de brillo y de color entre los colores usados para imágenes y fondo? ¿Considera adecuado que el usuario pueda guardar los datos de una pantalla con los datos incompletos? ¿El usuario tiene la opción de hacer clic directamente sobre una caja de texto? ¿Cada ícono está resaltado con respecto al color de su fondo? ¿La función de ayuda es fácil de ver por el usuario? ¿La información de la ayuda es comprensible? ¿El registro de datos en las pantallas es fácil de aprender y usar? ¿Considera que el ambiente de los íconos es ordenado? ¿El diseño de los íconos es adecuado para la aplicación? Estas preguntas fueron basadas en las recomendaciones de Jacob Nielsen (1994).

\section{Metodología}

\section{Población y muestra}

Se realizó un estudio bajo el enfoque o paradigma cuantitativo. De acuerdo con Hernández, Fernández y Baptista (2014), es secuencial y probatorio: cada etapa precede a la siguiente. Parte de una idea, que va delimitándose, y de la cual se derivan objetivos y preguntas de investigación. Se revisa la literatura y se construye un marco o una perspectiva teórica. De las preguntas se establecen hipótesis y se determinan variables. A continuación, se desarrolla un plan para probarlas (diseño). Enseguida, se miden las variables en un determinado contexto y se analizan las mediciones obtenidas (con frecuencia utilizando métodos estadísticos). Por último, se establece una serie de conclusiones respecto de la(s) hipótesis.

El alcance que se eligió para realizar esta investigación fue el descriptivo, debido a que se expusieron las variables de usabilidad que sirven para evaluar una aplicación móvil. Por lo tanto, no se busca relacionar variables ni explorar un tema poco conocido. 


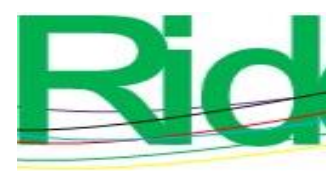

랑

Revista Iberoamericana para la Investigación y el Desarrollo Educativo ISSN $2007-7467$

El diseño fue no experimental, ya que no se recurrió a la manipulación de variables, tal como lo explican Hernández et al. (2014). Asimismo, los diseños de investigación transaccionales o transversales recolectan datos en un solo momento, en un tiempo único. (Hernández et al., 2014). El presente estudio tuvo un diseño transaccional.

Se consideró como población a los estudiantes de la carrera de ingeniería Informática. Para la determinación del tamaño de la muestra, se utilizó la fórmula de muestreo aleatorio estratificado (Mellado, 2009). Dicha fórmula se describe a continuación:

$$
\frac{n}{N}=\frac{n 1}{N 1}=\frac{n 2}{N 2}=\frac{n 3}{N 3}
$$

Dónde:

$$
\begin{array}{ll}
\text { - } & n=\text { muestra } \\
\text { - } & N=\text { total de población, } \\
\text { - } & n 1=\text { estrato } 1, \\
\text { - } & n 2=\text { estrato } 2 \mathrm{y} \\
\text { - } & n 3=\text { estrato } 3 .
\end{array}
$$

Usando la fórmula, el total de la muestra fue de 68 alumnos de tres salones diferentes: 22 alumnos de segundo semestre, 23 de sexto y 23 de octavo semestre.

\section{Método para el acopio de información}

Primero se administró un cuestionario general a todas las carreras del Instituto Tecnológico de Tizimín (ingeniería Informática, ingeniería en Agronomía, ingeniería en Gestión Empresarial, licenciatura en Administración, licenciatura en Biología). Resultaron un total de 200 participantes.

El instrumento estuvo conformado de 18 preguntas de opción múltiple y preguntas abiertas, con la finalidad de conocer acerca de la manera en la que los estudiantes utilizan sus dispositivos móviles, y así, poder determinar si estarían de acuerdo en incluir una aplicación móvil colaborativa dentro de sus actividades académicas.

Una vez que se obtuvieron esos datos, se seleccionó la muestra con la fórmula ya descrita y se les administró un cuestionario de heurística de evaluación para la aplicación móvil. Dicho instrumento constó de 30 preguntas de opción múltiple. 


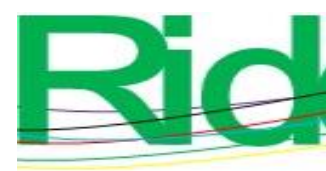

,

Revista Iberoamericana para la Investigación y el Desarrollo Educativo

ISSN $2007-7467$

La información se recolectó y ordenó a través de archivos en la computadora. Se utilizó el software estadístico SPSS, que es un software de análisis estadístico. De igual manera, fue necesario el programa Excel de Microsoft para representar la información en gráficas fáciles de interpretar.

\section{Resultados}

A continuación, se presentan los resultados del estudio.

En la tabla 1 se presentan las variables de usabilidad que permitieron realizar la evaluación de la aplicación móvil.

Tabla 1. Variables de usabilidad para evaluación de aplicación móvil

\begin{tabular}{|c|c|c|}
\hline Variables & Sí & No \\
\hline $\begin{array}{l}\text { 1) ¿Prefiere las aplicaciones de paga o } \\
\text { gratuita? }\end{array}$ & $\begin{array}{l}\text { Las apps } \\
\text { gratuitas } \\
\quad 95.5\end{array}$ & $\begin{array}{l}\text { Las apps de } \\
\text { paga } \\
\quad 4.5\end{array}$ \\
\hline 2) ¿Qué sistema operativo tienes? & $\begin{array}{c}\text { Android } \\
95.5\end{array}$ & $\begin{array}{l}\mathrm{iOS} \\
4.5\end{array}$ \\
\hline $\begin{array}{l}\text { 3) ¿Consideras que las apps te } \\
\text { permitirían ser más eficiente en tus } \\
\text { labores educativas diarias? }\end{array}$ & 93.2 & 6.8 \\
\hline $\begin{array}{l}\text { 4) ¿Cuál es el mayor uso que le da a } \\
\text { las apps? }\end{array}$ & 50.1 & 40.9 \\
\hline $\begin{array}{l}\text { 5) ¿Te gustaría que sean más prácticos } \\
\text { los trabajos en equipo con ayuda de } \\
\text { una aplicación móvil? }\end{array}$ & 95.5 & 4.5 \\
\hline $\begin{array}{l}\text { 6) En promedio, ¿cuánto tiempo pasas } \\
\text { interactuando con tu dispositivo } \\
\text { móvil? }\end{array}$ & 50 & 50 \\
\hline $\begin{array}{l}\text { 7) ¿Habías escuchado con } \\
\text { anterioridad sobre el concepto de } \\
\text { aprendizaje móvil? }\end{array}$ & 54.5 & 45.5 \\
\hline
\end{tabular}



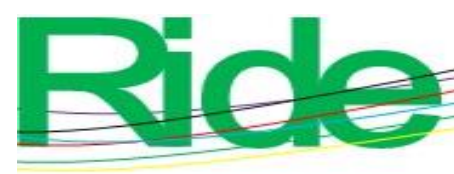

Revista Iberoamericana para la Investigación y el Desarrollo Educativo ISSN 2007 - 7467

Figura 2. Estándares de usabilidad de la aplicación móvil colaborativa

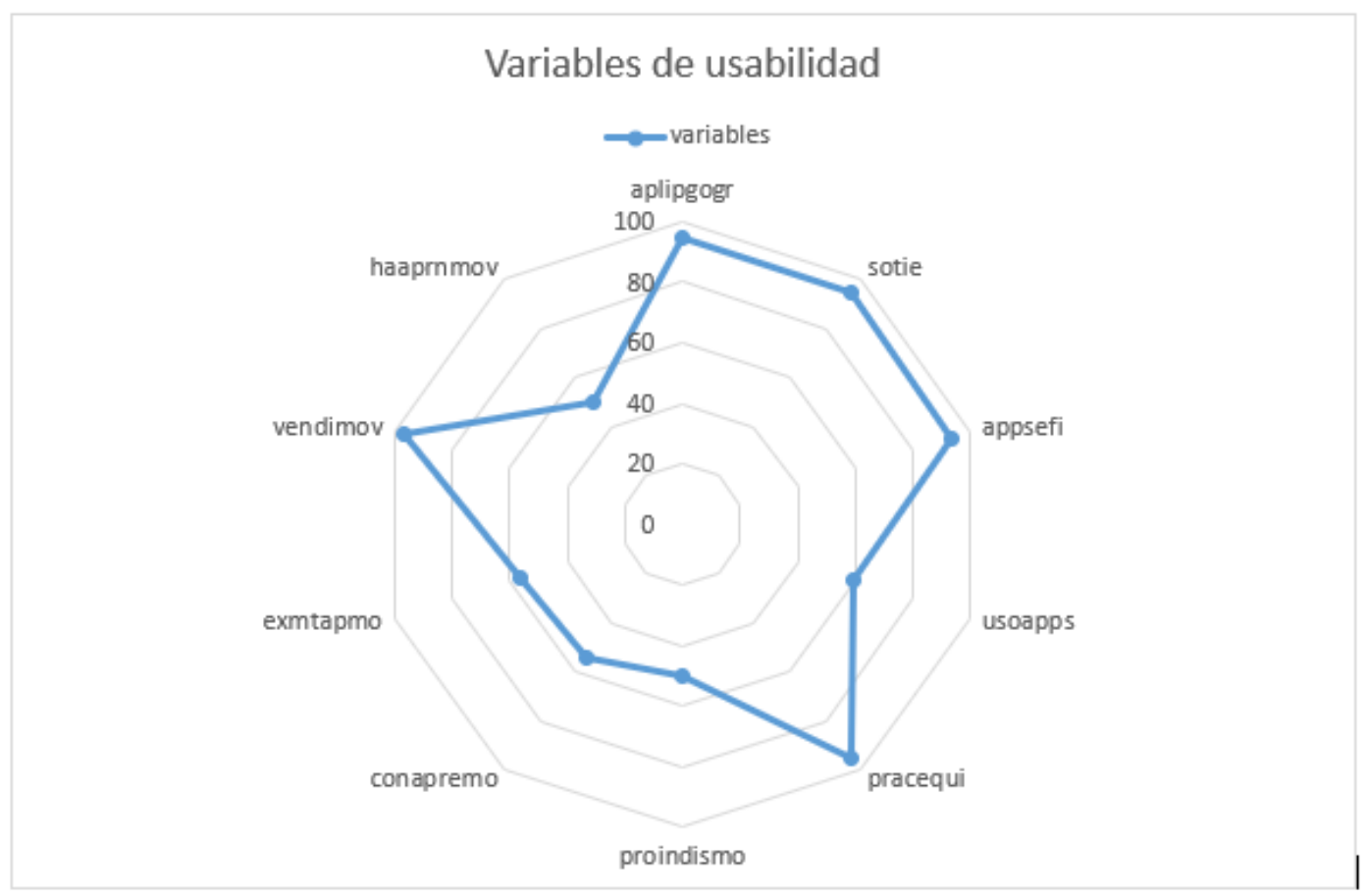

Fuente: Elaboración propia

En la figura 2 se muestran las variables que se tomaron en cuenta para la evaluación del funcionamiento y usabilidad de la aplicación móvil colaborativa. Algunos puntos para destacar se enlistan a continuación:

- $\quad$ Variable uno: $95.5 \%$ prefiere que la app sea gratuita al momento de salir al mercado y $4.5 \%$ que sea una aplicación de paga. Ese fue el resultado obtenido de la encuesta hecha a los estudiantes.

- Variable dos: la mayoría de los estudiantes encuestados tiene un celular con sistema operativo Android (95.5\%), lo cual es favorable, porque sí podrán instalar la aplicación; un porcentaje mínimo, $4.5 \%$, indica que tienen celular con sistema operativo iOS. En suma, es viable la instalación de la aplicación para la mayoría de los estudiantes.

- $\quad$ Variable tres: 93.2 \% afirma que la app le será de mucha ayuda en el área educativa y $6.8 \%$ no la encontró útil.

- $\quad$ Variable cuatro: $50.1 \%$ afirma utilizar más las apps para la educación y 40.9 $\%$ para otras actividades.

- Variable cinco: $95.5 \%$ considera que prefiere que sean más prácticos los trabajos en equipo con el uso del celular, y $4.5 \%$ consideró que no, que prefieren el método común. 

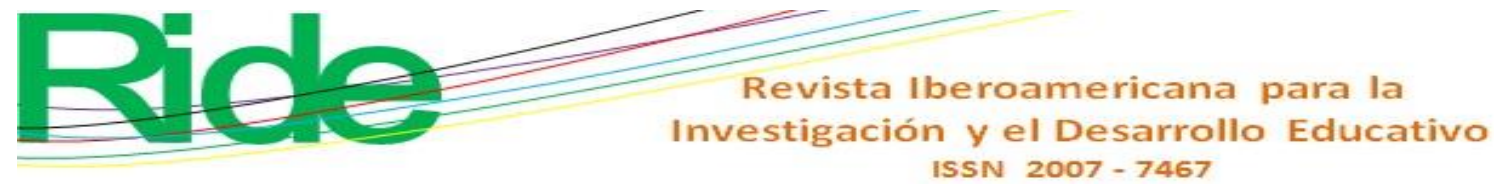

- Variable seis: en promedio $50 \%$ de los estudiantes pasa de cuatro horas a seis horas diarias en el celular, y la otra mitad lo hace por un tiempo menor.

- Variable siete: $54.5 \%$ aseguró que ha escuchado algo relacionado con el concepto de aprendizaje móvil y el $45.5 \%$ lo desconocía totalmente.

- Variable ocho: complementado la variable anterior, $43.2 \%$ expresó tener experiencia trabajando con esta metodología, y $56.8 \%$ negó haber trabajado en el pasado con ella.

- Variable nueve: $97.7 \%$ recomienda implementar el uso de celulares en actividades para un mejor aprendizaje; $2.3 \%$ no lo recomienda.

- Variable 10: la mitad de los involucrados mencionó que le gustaría realizar un proyecto relacionado con la educación y las aplicaciones móviles y la otra no tiene interés alguno en realizarlo.

Como se puede visualizar, el resultado final obtenido de las variables implementadas para la evaluación de la aplicación móvil indica que 72.52 \% de los participantes afirma que la aplicación cumple de manera positiva con las variables de usabilidad descritas, ya que contiene lo necesario para un correcto funcionamiento en cuanto a diseño y usabilidad. Contrariamente, el porcentaje restante, $26.58 \%$, está en desacuerdo, es decir, no considera que cumpla con los requerimientos.

\section{Variables de viabilidad}

Figura 3. Estándares de viabilidad de la aplicación móvil colaborativa

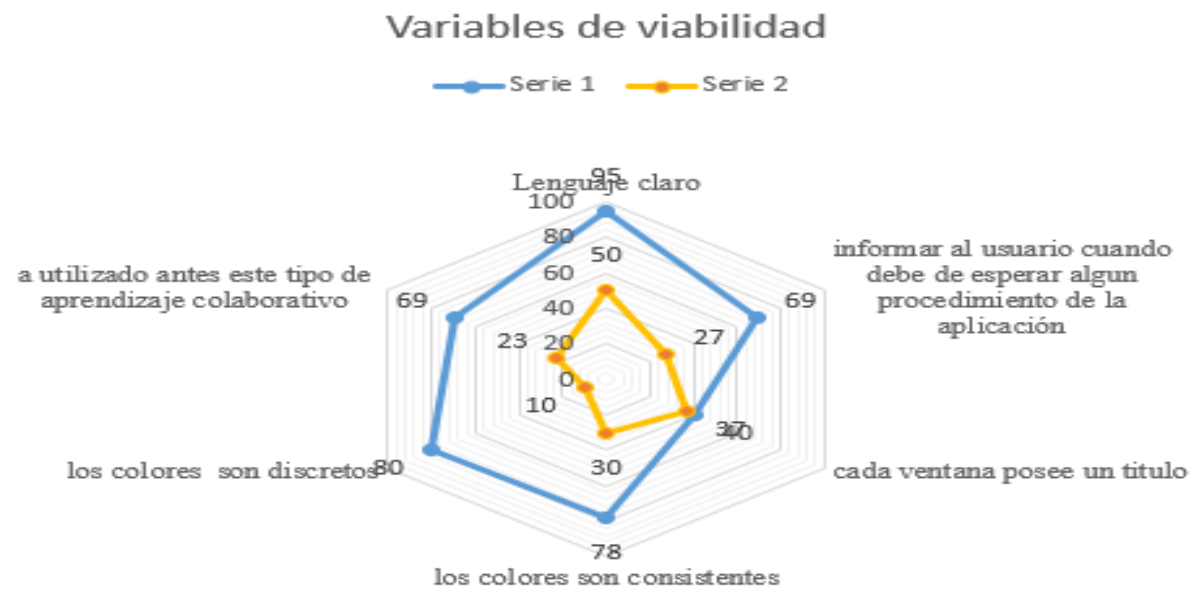

Fuente: Elaboración propia 

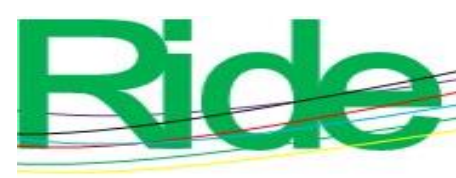

Revista Iberoamericana para la Investigación y el Desarrollo Educativo ISSN 2007 - 7467

En la figura 3 se muestran las variables que se tomaron en cuenta para la evaluación de la viabilidad de la aplicación móvil colaborativa. Los siguientes puntos resumen dicha gráfica

- Variable uno: $100 \%$ de los participantes considera que la aplicación tiene un lenguaje entendible.

- $\quad$ Variable dos: $69 \%$ prefiere que la aplicación informe al usuario cuando debe esperar algún procedimiento.

- $\quad$ Variable tres: $37 \%$ respaldó la idea de que todas las ventanas deben contener título, si bien hay ventanas que no lo pueden hacer porque son emergentes.

- Variable cuatro: de acuerdo con $78 \%$ de los participantes, los colores son consistentes en toda la aplicación.

- $\quad$ La variable cinco: del total de la muestra, 80 \% consideró idóneos los colores de la aplicación móvil.

- $\quad$ La variable seis: $69 \%$ de los participantes ha recurrido antes a este tipo de aprendizaje colaborativo.

\section{Discusión}

En esta investigación se encontró que la aplicación cumple en su mayoría con los parámetros, así como con las variables de usabilidad evaluadas, las cuales se basaron en la información sugerida por el autor Jacob Nielsen (1994). En suma, funciona de manera correcta y puede ser usada fácilmente.

Una debilidad de la aplicación respecto a la variable de uso y documentación fue que no cuenta con esta opción, lo cual dificulta al usuario, si tuviera alguna duda acerca del funcionamiento.

Por lo tanto, las respuestas dadas por los usuarios que fungieron como evaluadores de la aplicación móvil indican que esta cumple con las características de uso y el propósito para el que fue diseñado: una herramienta para el aprendizaje colaborativo en el aula.

Los resultados son coincidentes con otros estudios. Por ejemplo, el de Lapuente (2013), quien, al evaluar la usabilidad de una aplicación móvil para sistemas Android diseñada para ayudar a pacientes con enfermedades crónicas a seguir las pautas de medicación, encontró que el criterio funcional prevaleció en la aplicación. Dicho estudio demuestra, además, que las aplicaciones móviles se están usando en todos los contextos. 


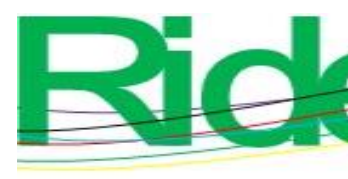

Revista Iberoamericana para la Investigación y el Desarrollo Educativo ISSN 2007 - 7467

De igual manera, Bojórquez (2011) realizó un estudio que consistió en evaluar la usabilidad de una aplicación móvil que provea información del seguimiento del aprendizaje en los estudiantes, cuyos resultados fueron aprobatorios, la aplicación funcionó, aunque algunas sugerencias fueron la de incluir una ayuda inicial sobre el uso del sistema y señalar de manera visual y colorida los mensajes de alerta y errores.

Los resultados de estas investigaciones coinciden con lo encontrado en el presente estudio, aunque existen diferencias debido al contexto en el que las pruebas fueron desarrolladas y las metodologías utilizadas. Teniendo en cuenta lo anterior, en el mismo instituto y usando las mismas preguntas basadas en la usabilidad de Jacob Nielsen (1994), se probó una aplicación referente a la trazabilidad del ganado. Los resultados fueron de aceptación, lo cual llevó a utilizar la misma metodología.

\section{Conclusiones}

En los últimos años, diversos dispositivos electrónicos, laptops, tabletas, teléfonos celulares, se han integrado a las aulas educativas de los distintos niveles, lo cual ha dado lugar a un cambio importante de estrategias de enseñanza. Cada vez más softwares y aplicaciones móviles se han incluido con el fin de hacer el aprendizaje más significativo para el estudiantado.

El presente estudio de investigación se planteó con el fin de verificar el grado de usabilidad de una aplicación móvil colaborativa. Los resultados obtenidos fueron favorables, ya que la mayoría de los participantes de la prueba de la aplicación coincidió con los parámetros de usabilidad. Incluso los aspectos que no lograron un puntaje óptimo sirvieron para trazar y realizar modificaciones al sistema.

Con respecto a los objetivos planteados, se puede concluir que la evaluación de usabilidad realizada permitió enriquecer los conocimientos en relación con los puntos que se deben tomar en cuenta a la hora de medir el grado de usabilidad de un software. Dado que el objetivo principal era conocer el nivel de usabilidad de una aplicación móvil, la aplicación se utilizó en las aulas de la carrera de ingeniería Informática del Instituto Tecnológico de Tizimín; así, se pudo realizar la aplicación de la heurística diseñada y de esta forma observar los resultados de cada estudiante.

Como conclusión habría que decir que las pruebas realizadas fueron un éxito, ya que se cumplió con la mayoría de los parámetros establecidos de usabilidad, sin embargo, una de las variables no se cumplió de manera satisfactoria, y esto dificulta un poco el uso de la 


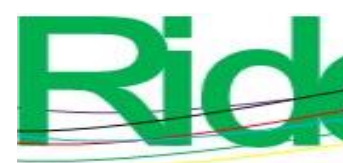

Revista Iberoamericana para la
Investigación y el Desarrollo Educativo ISSN $2007-7467$

aplicación. La única variable de usabilidad que no se cumplió de manera satisfactoria fue la número 10, referente a la ayuda y documentación, esto debido a que la aplicación no cuenta con ningún manual de uso o sección de apoyo.

Como propuesta se puede realizar un manual de uso que incluya un apartado de preguntas frecuentes, esto con la finalidad de tener una guía que sirva de introducción a los nuevos usuarios y puedan usar satisfactoriamente esta herramienta colaborativa.

\section{Futuras líneas de investigación}

El presente artículo proporciona una pauta para continuar con futuras líneas de investigación en temas relacionados con la educación y la búsqueda de mejoras en el desempeño académico de los alumnos al implementar herramientas de aprendizaje colaborativo, así como en el apoyo en la enseñanza y las estrategias que los docentes aplican durante sus clases mediante aplicaciones virtuales de aprendizaje.

Aunado a lo anterior, este artículo da la pauta para que el proyecto continúe con la modificación de los puntos que salieron con menor calificación y así incrementar el grado de usabilidad. También, para implementar un estudio de sistemas expertos que puedan analizar los $\log s$ generados por la aplicación y con ello poder calificar de forma automática a los estudiantes que colaboraron, lo que llevaría a otro estudio, que sería verificar si la calificación otorgada toma en cuenta realmente la interacción entre los estudiantes de forma colaborativa.

Finalmente, da pie a la elaboración de nuevos proyectos de investigación con herramientas de aprendizaje colaborativo, así como a la modificación de dichas herramientas con las que ya se han trabajado, o a realizar comparaciones con otras, siempre con el objetivo de obtener mejores resultados en términos de usabilidad. 


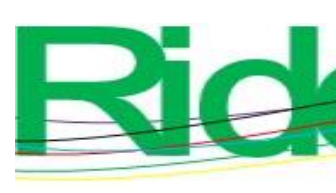

Revista Iberoamericana para la Investigación y el Desarrollo Educativo ISSN $2007-7467$

\section{Referencias}

Bojórquez, E. (2011). Evaluación de la usabilidad de un sistema informático móvil para asesorías de asignaturas en la Universidad Autónoma Indígena de México. $R a$ Ximhai, 7(1), 33-39.

Enriquez, J. G. y Casas, S. I. (2013). Usabilidad en aplicaciones móviles. Informes Científicos Técnicos UNPA, 5(2), 25-47.

Escofet, A. y Marimon, M. (2012). Indicadores de análisis de procesos de aprendizaje colaborativo en entornos virtuales de formación universitaria. Enseñanza \& Teaching, 30(1), 85-114.

Ferré, G. (2000). Principios básicos de usabilidad para ingenieros software. Ponencia presentada en las V Jornadas de Ingeniería del Software y Bases de Datos. Valladolid, del 8 al 10 de noviembre de 2000.

Frøkjær, E., Hertzum, M. and Hornbæk, K. (2000). Measuring Usability Are Effectiveness, Efficiency and Satisfaction Really Correlated? Paper presented at the CHI 2000: Human Factors in Computing Systems. The Hague, April 1-6, 2000.

Hernández, R., Fernández, C. y Baptista, P. (2014). Metodología de la investigación. México: McGraw-Hill Education.

Institute of Electrical and Electronics Engineers [IEEE]. (1998). 1061-1998 - IEEE Standard for a Software Quality Metrics Methodology. New Jersey, United States: Institute of Electrical and Electronics Engineers.

Lamí, L. E., Pérez, M. G. y Rodríguez del Rey, M. E. (2016). Las herramientas de comunicación sincrónica y asincrónica en la clase presencial. Conrado, 12(56), 8489. Recuperado de http://conrado.ucf.edu.cu/.

Lapuente, E. (2013). Implementación y evaluación de la usabilidad de una aplicación para sistemas Android de cumplimiento de pauta de medicación en pacientes con enfermedades crónicas. (Tesis de maestría). Universidad de Valladolid, Valladolid.

Mellado, B. (2009). Las TIC en educación. Departamento de Estadística y Cálculo.

Nielsen, J. (1994). Evaluación heurística. Nueva York, Estados Unidos: John Wiley \& Sons.

Quisi, D. (2012). Diseño e implementación de una aplicación para dispositivos android en el marco del Proyecto pequeñas y pequeños científicos de la Universidad Politécnica Salesiana. (Tesis de licenciatura). Universidad Politécnica Salesiana, Cuenca.

Seffah, A., Donyaee, M., Kline, R and Padda, H. (2006). Usability measurement and metrics: A consolidated model. Software Quality Journal, 14, 159-178. 


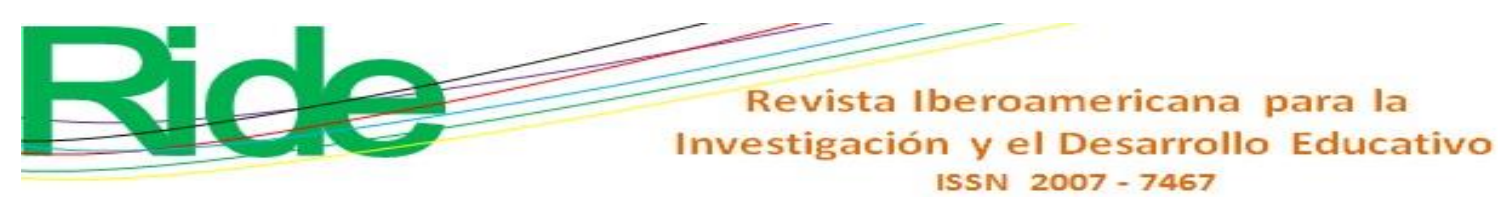

Stufflebeam, D y Shinkfield, A. (1995). Evaluación sistemática: guía teórica y práctica. España: Paidós/Ministerio de Educación y Ciencia.

Tardáguila, C. (2009). Dispositivos móviles y multimedia. Mosaic. 